\title{
A Plethora of Actions of Curcumin - A Magical Agent for Treatment of Wide Range of Diseases Varying from Neuroinflammatory Disease (AD, PD)-IBD to DM and CVD, NAFLD, NASH Along with Various Cancers - A Systematic Review
}

\author{
Kulvinder Kochar Kaur ${ }^{1 *}$, Gautam Allahbadia ${ }^{2}$ and Mandeep Singh ${ }^{3}$ \\ ${ }^{1}$ Scientific Director, Dr Kulvinder Kaur Centre for Human Reproduction, Jalandhar, \\ Punjab, India \\ ${ }^{2}$ Scientific Director, Rotunda - A Centre for Human Reproduction, Mumbai, India \\ ${ }^{3}$ Consultant Neurologist, Swami Satyan and Hospital, Jalandhar, Punjab, India
}

*Corresponding Author: Kulvinder Kochar Kaur, Scientific Director, Dr Kulvinder

Kaur Centre for Human Reproduction, Jalandhar, Punjab, India.
Received: November 22, 2021

Published: January 17, 2022

(C) All rights are reserved by Kulvinder

Kochar Kaur., et al.

\begin{abstract}
Curcumin represents a yellow polyphenolic pigment derived from the plant Curcuma longa (turmeric) rhizome that has been put in utilization over centuries for cuisine art besides coloring of foods, as a constituent of separate medicinal formulations that has been used over and over in Ayurveda along with Chinese medicine. In the current decades it has been exhaustively evaluated for its biological actions. Thus here we conducted a systematic review with the utilization of search engines like pubmed, google scholar, web of science, Cochrane library from 1900 till date with the utilization of MeSH terms Curcumin; Neuroinflammation; Alzheimer's disease (AD); parkinson's disease (PD); antioxidant actions; other anti-inflammatory actions; cardio protection; hepatoprotection in liver fibrosia; non alcoholic fatty liver disease (NAFLD); non alcoholic steatohepatitis (NASH); Anti cancer actions. We observed a total of over 3000 articles but selected only 53 articles for this review with the journals constraints of up to 50 references. No metaanalysis was done. Here we have reviewed in detail the mechanism of action of Curcumin in various above mentioned diseases along with transcription factors involved. Recently even in COVID its use has got documented. Although use in Alzheimer's disease studied extensively human studies are still battling to get a concrete answer. In case of cancers, usually it is a good coagent with other chemotherapeutic drugs, might hamper human papilloma virus (HPV) infection as well as avoid cancer cervix generation. In case of NASH, or liver cirrhosis its use with beta blockers might aid in conversion back towards normalcy.
\end{abstract}

Keywords: Curcumin; Anti Inflammatory; Anti Oxidant; Neuro Shielding; Cardio Shielding; Hepatoprotection; Anti Cancer Actions

\section{Introduction}

Curcumin represents a yellow polyphenolic pigment derived from the plant Curcuma longa (turmeric) rhizome has got utilized for over centuries, for culinary along with food colouring characteristics, in addition to a component of different medicines, that has got extensively used in Aurveda as well as Chinese medicines. In the current decades their biological actions have got evaluated in a lot of details. Here aim of the review is to provide a deep ex- planation of Curcumin applied to food as well as biotechnological industries, besides health facilitation as well as disease avoidance with specific highlight of its anti oxidant, anti-inflammatory, neuroshielding, anticancer, hapatoprotection, as well as cardioshielding actions. Further its bioavailability, bio effectiveness as well as safety parameters along with quality properties of Curcumin are further tackled. Lastly multidimensional uses of Curcumin, lucrativeness of food looking attractive. 
A Plethora of Actions of Curcumin - A Magical Agent for Treatment of Wide Range of Diseases Varying from Neuroinflammatory Disease (AD, PD)-IBD to DM and CVD, NAFLD, NASH Along with Various Cancers - A Systematic Review

Curcumin species- small general detail of curcumin

For details of curcuma genus [2].

\section{Historical aspect of Curcumin}

Started from far eastern medicines 5000 (Ayurveda) as well as 2000 yrs (Atharveda) ago respectively. Curcuma longa (C. longa) possesses various Curcuminoids, despite Curcumin was observed to be the maximum active (init identified in 1815 (vogel-pelletier-1815), along with the purified crystalline compound-detected in 1870. Its structure was initially illustrated by polish scientists (Figure 1) [1]. Despite generally Curcumin talks about -1,7-bis(4hydrocxy-3methoxy phenyl) 1.6 heptadiene,3,5dione, it is also called "Curcumin1". That is Curcumin-is adiferuloyl methane see figure 1 for structure [2] for more details.

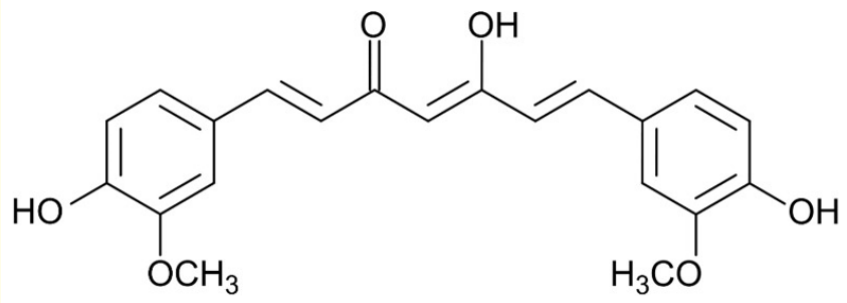

Figure 1: Chemical structure of curcumin.

Curcumin on health propagation as well as disease avoidance

Lot of actions on Health Propagation as well as disease avoidance seen with curcumin as well as derivatives. A bibliometric evaluation done by Yeung., et al. [3] documented that the USA, China, India, Japan as well as South Korea have done maximum scientific work observed on Curcumin bioactive actions with the maximum concentrations on their anti cancer, inflammatory, with anti oxidant capacity, as revealed by Xu., et al. (2018) [4]. Here preclinical as well as clinical results associated with Curcumin bioactive actions is detailed in brief with mechanism of action discussed.

\section{Anti oxidant action}

The Curcumin anti oxidant actions have been the mechanism evaluated in literature. Lot of in vivo, as well as vitro studies have got performed, in addition to the anti oxidant action has been correlated with its chemical structure, that includes carbon- carbon double bonds, $\beta$ diketo group as well as phenyl rings with hydroxyl along with o-methoxy groups [5]. Numerous modes can reason out the anti oxidant action in the form of free binding radicals, hydrogen atom donors and electron donors for neutralization of free radicals. For this purpose utilization of Laser flash photolysis, besides pulse radiolysis were done for unraveling the mode of action of Curcumin' anti oxidant actions [6].

Curcumin' has the capacity to facilitate antioxidant actions by scavenging a lot of Reactive oxygen species (ROS) in the form of superoxide radicals, hydrogen peroxide $\left(\mathrm{H}_{2} \mathrm{O}_{2}\right)$ as well as nitric oxide (NO) radicals in addition to hampering lipid peroxidation [7]. This action is secondary to enhancement of a lot of anti oxidant enzymes action like super oxide dismutase (SOD), CAT, glutathione peroxidase (GPx), as well as a $\mathrm{OH}-1$. Further Curcumin can result in escalation of GSH amounts by upregulation of glutathione transferase along with their mRNA. Furthermore Curcumin can hamper ROS producing enzyme like lipoxygenase (LOX), COX as well as xanthine oxidase. It is also believed to be a chain breaking anti oxidant in view of its lipophilic nature, acting basically as a peroxy radicals scavenger [5].

\section{Anti inflammatory actions}

Numerous in vivo, along with in vitro studies have demonstrated, that Curcumin possesses big capacity for therapy of numerous inflammatory diseases $[8,9]$. It got documented that Curcumin can i) hamper proinflammatory factors, transcription factors like nuclear factor $\mathrm{K}(\mathrm{NFKB})$ as well as activator protein1 (AP1). ii) Result in reduction of proinflammatory cytokines, like tumor necrosis factor alpha (TNF $\alpha$ ) along with interleukin-1b (IL-1b) IL-2, IL-6. IL-8, macrophage inflammatory protein $1-\alpha$ (MIP1 $\alpha$ ), monocyte chemoattractant protein (MCP1), C Reactive Protein (CRP), as well as prostaglandin E2 (PGE2). iii) down regulation of lipoxygenase along with cyclooxygenase $2\left(\mathrm{COX}_{2}\right)$ as well as iv) Inhibition of mitogen activated protein kinase (MAPK) in addition to pathways implicated in nitric oxide synthase (NOS) [10]. Figure 2 illustrates the anti inflammatory modes.

Conversely, with the knowledge that Oxidative stress (OS) triggers chronic inflammation, an intricate association among anti oxidant molecules as well as its anti inflammatory prospects has become escalatingly apparent. That way Curcumin further has the capacity of modulation of NFKB expression. Actually the pathway activation results in proinflammatory cytokines generation, like 
[IL-1, IL-2, IL-6. IL-8 along with TNF $\alpha$ that have been accepted to be stimulating proinflammatory signaling pathway. Additionally, Curcumin result in reduction of Oxidative stress in addition to inflammation via the Nrf2 as well as NFKB pathway. The COX pathway results in the transformation of arachidonic acid into prostaglandins along with thromboxane, with two COX isoenzymes (COX1 as well as COX2) being implicated. Specifically, COX2 gets stimulated by different cytokines in addition to tumor promoters, hence intricately associated with inflammation along with carcinogenesis, with a lot of studies documenting that Curcumin can hamper the stimulation of COX2 gene expression [11].

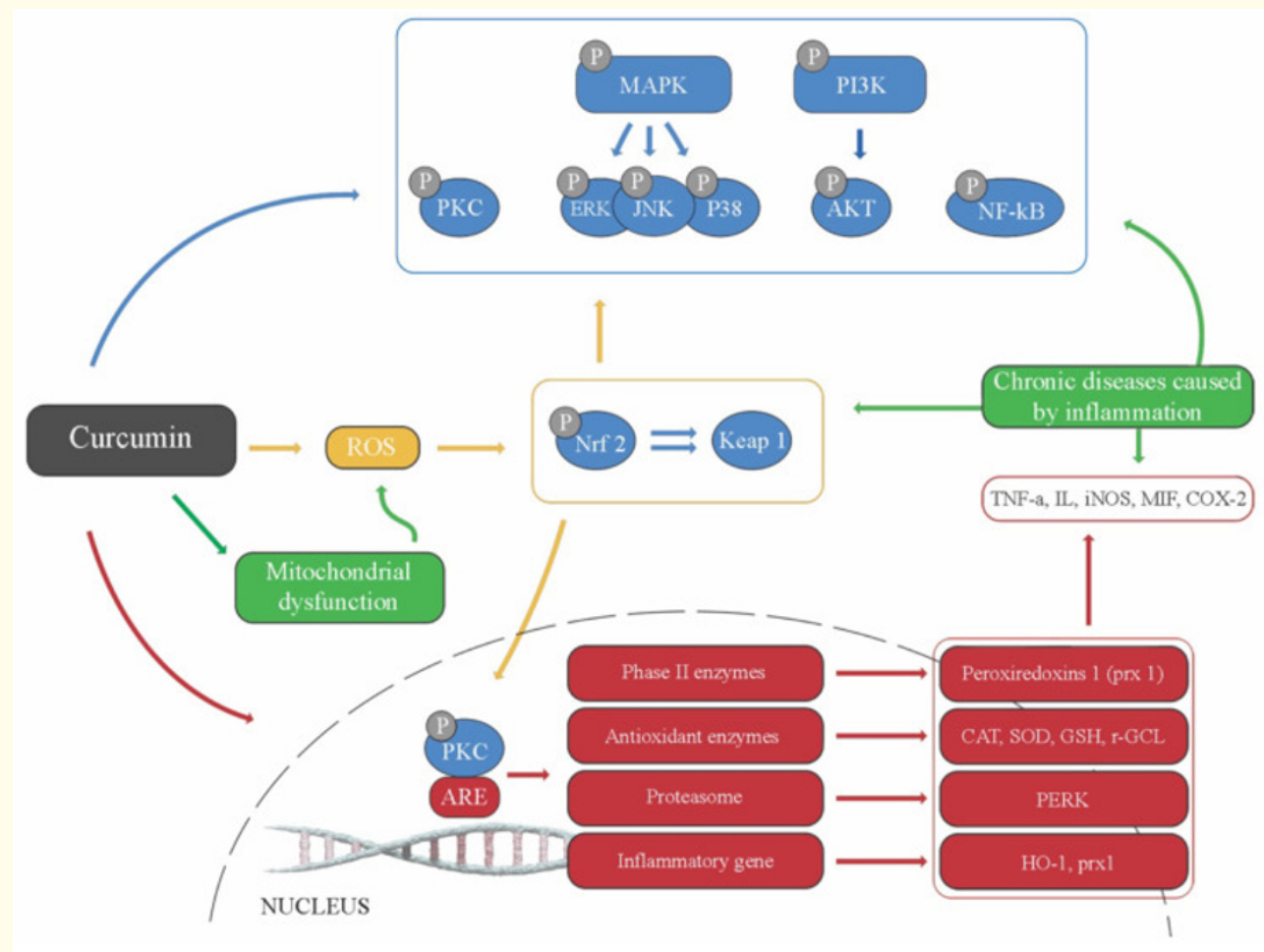

Figure 2: Courtesy ref no2 - Curcumin anti-inflammatory mechanisms.

\section{Neuroshielding actions}

Neurodegenerative conditions like Parkinson's diseases (PD) as well as Alzheimer's disease (AD), major depression as well as epilepsy implicate millions of people throughout the world, with escalated incidence rate. Neuroinflammation represents a chronic inflammation which results in neuronal metabolism alterations which cause neuronal breakdown. In Neuroinflammatory conditions, the neuronal demise gets escalated by microglia as well as astrocytes activation. Astrocytes are the ones that result in proinflammatory cytokines liberation like TNF $\alpha$ as well as IL-1. Depending on the present studies, Curcumin utilization in the form of a promising potential treatment agent for separate neurological conditions like dementia, AD, PD, multiple sclerosis (MS), along with huntington's disease (HD), secondary to its anti oxidant, anti inflammatory as well as anti protein accumulating capacities [12,13]. It has been demonstrated that Curcumin blocks the inflammatory cytokines along with PG generation in case of activated microglia as well as astrocytes $[11,14]$. It further causes reduction in the generation of TNF $\alpha$, IL-1 $\beta$, MIP-1 $\beta$, MCP1, along with IL-8 in microglia as well as astrocytes cells [15], figure 3 illustrates these aspects in small detail. 


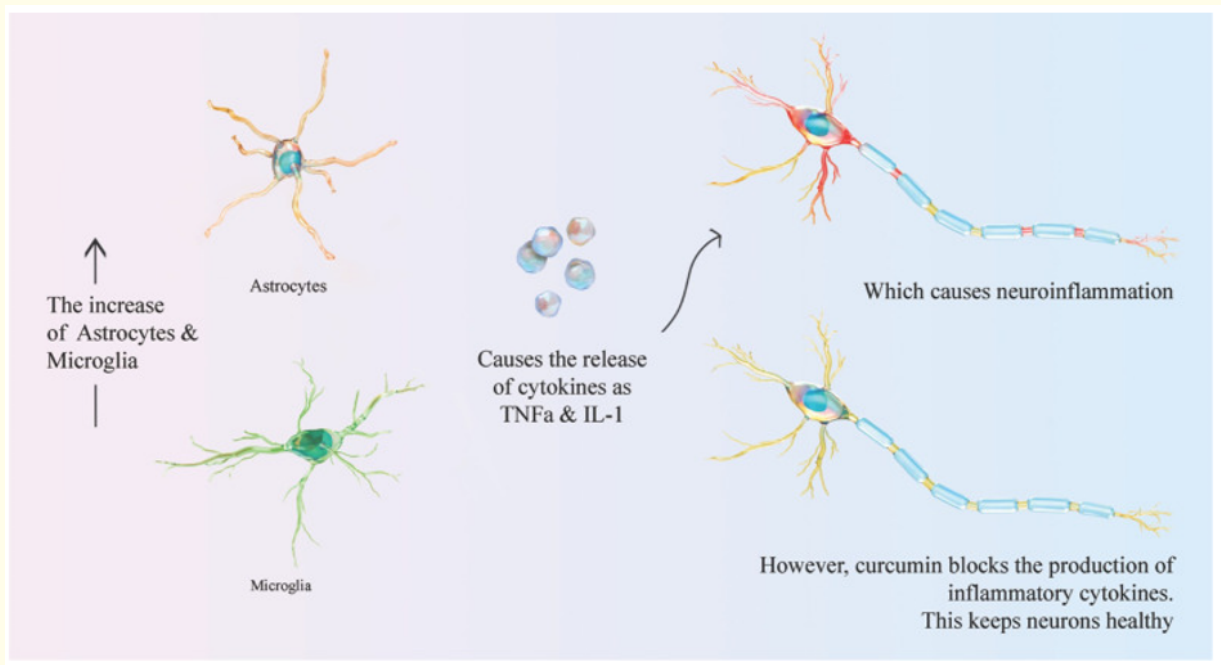

Figure 3: Courtesy ref no-2 - Neuroprotective mechanisms of curcumin in treating Parkinson's disease.

In case of $\mathrm{AD}$, the major aetiology is the amyloid $\beta(\mathrm{A} \beta)$ peptide plaques getting deposited, that is secondary to microgliosis in the brain. Curcumin was observed to decrease AD symptomatology $[16,17]$ by i) hampering the $A \beta$ peptide generation by changing the amyloid precursor protein trading. ii) binding the $A \beta$ peptides as well as implicating their collection. iii) ameliorating the tau hyper phosphorylation in addition to escalation of its clearing. iv) decreased $A \beta$ stimulated toxicity via the hampering of JNK3 phosphorylation. $v$ ) reduction in cholesterol amount that decreases AD risk. vi) shielding the Blood Brain Barrier (BBB) by up regulation of $\mathrm{OH}-1$ expression. vii) hampering acetyl cholinesterase. viii) having a part in cell signaling via activation of Wnt pathways as well. ix) causing reduction in inflammation in addition to oxidative damage. Akin to that certain studies have demonstrated that Curcumin has the capacity of treatment of PD. Figure 4 demonstrates the Neuroshielding mode of Curcumin in treatment of PD.

In case of MS, an autoimmune inflammatory disorder which primarily influences young adults in addition to women via demyelinating lesions. ii) Curcumin has further demonstrated Neuroshielding actions via desperate modes that are anti oxidant, antiinflammatory as well as anti proliferation modes. Further Curcumin possessed the capacity of modulation of various molecular targets, like the transcriptional factors Nrf2, NFkB, AP1, Signal Transducers and Activators of transcription (STAT1,-3.-4) enzymes (COX2, INOS, $\mathrm{OH}-1$, LOX, XO) inflammatory cytokines (chemokine ligand, TNF $\alpha$ ) as well as interleukin), proteins (caspase-3,-9,Bcell lymphoma2 [Bcl2], PG, CRP, myosin light chain], protein kinase (AK, JNK, JAK, MAPK) and growth factor as well as receptors (toll like receptors [TLRs], chemokine receptor, transforming growth factor $\alpha$ in addition to $\beta 1$ (TGF- $\alpha$, TGF- $\beta 1$ ] [18].

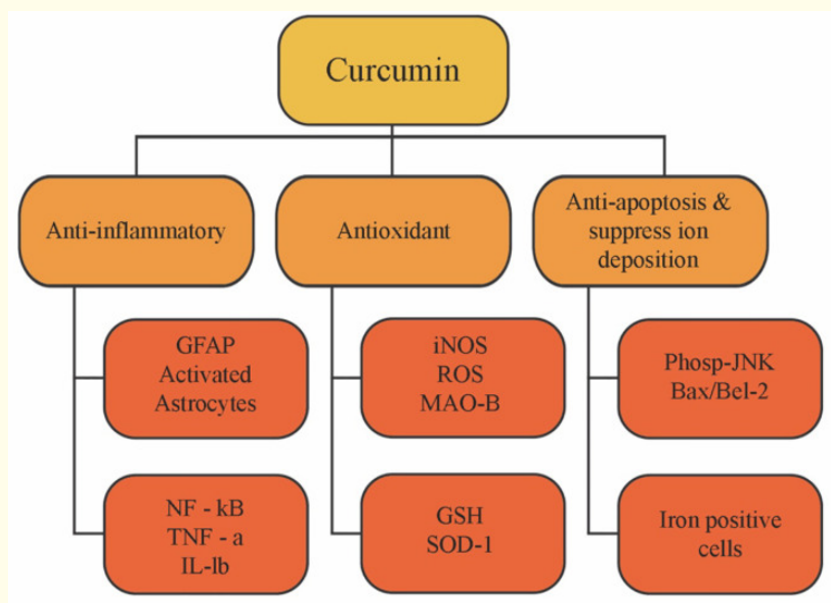

Figure 4: Courtesy ref no-2 - Neuroprotective mechanisms of curcumin in treating Parkinson's disease. 


\section{Anticancer actions}

Exhaustive preclinical studies can be observed in literature, evaluating Curcumin's Anticancer actions with escalating concentration being done on its mode of action. Curcumin (Chas been demonstrated to avoid carcinogenesis by influencing 2 events: angiogenesis as well as cancer cell growth. The modulation of various molecular targets via which Curcumin works is by up regulation or down regulation is illustrated in figure 5 .

The part of angiogenesis in Cancer is well accepted. Actually cancer cells can generate new blood vessels by stimulation of proangiogenic factors. Curcumin possesses antiangiogenic action by hampering proangiogenic factors stimulators like vascular endothelial growth factor (VEGF), basic fibroblast growth factor (bFGF). Curcumin has been demonstrated to result in down regulation of VEGF expression via NFKB, in addition to activator protein 1 (AP1), control, ameliorating IL-8 expression [19]. Astinfeshan., et al. [20], demonstrated that Curcumin has ability of hampering angiogenesis via VEGFR along with PI3K/Akt signaling pathway modulation. Furthermore it was demonstrated that Curcumin can cause down regulation of Matrix Metalloproteinases (MMP's), MMP2 as well as MMP9, besides up regulation of the tissue inhibitors of Matrix Metalloproteinases 1 (TIMP1), that guarantees stableness of the extracellular matrix [ECM] along with cohesion [19].

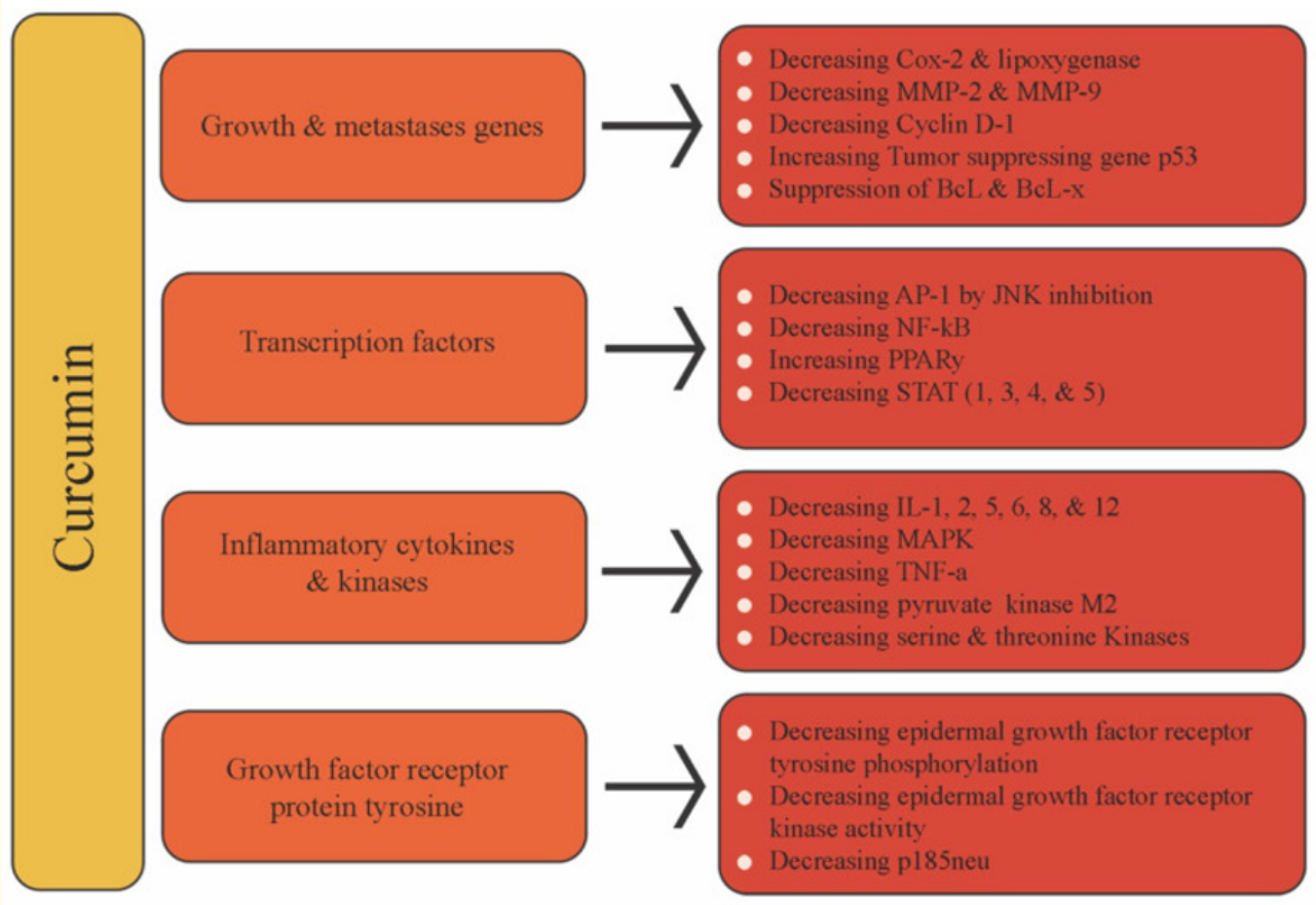

Figure 5: Courtesy ref no-2 - Curcumin molecular targets in cancer cells.

Curcumin stimulates apoptosis in Cancer Cells via p53-based pathway. p53 is understood to be one of maximum significant tumor suppression proteins, influencing cell proliferation, apoptosis along with DNA injury [21]. Various studies have documented a crosstalk among p53 as well as Cancer [15,22,23]. Ye., et al. [23], demonstrated proapoptotic actions of Curcumin that were based on miR 192-5p as well as miR 215, that stimulated p53 in non small cell lung cancer. Other studies have demonstrated that Curcumin stimulated apoptosis in p-53-independent apoptosis in human HT29colon cancer cells [24]. 
A Plethora of Actions of Curcumin - A Magical Agent for Treatment of Wide Range of Diseases Varying from Neuroinflammatory Disease (AD, PD)-IBD to DM and CVD, NAFLD, NASH Along with Various Cancers - A Systematic Review

Cyclin dependent kinase (CDK) are serine/threonine kinases which generate a complex with their respective Cyclin partner, hence regulation of cell cycle propagation. Changed CDK's expression are constantly seen in cancer cells. In a triple negative breast cancer cell line MDA MB231with Curcumin, Chiu and Su [25] demonstrated that Curcumin resulted in interference in CDK/Cyclin complexes, essential for cell cycle propagation in addition to cause down regulation of CyclinD1, needed for the propagation via the G1/S phase, besides whose high expression is correlated with maximum breast cancers, hence resulting in cell cycle arrest at G1.

Curcumin has been posited to possess great efficacy against cancers in which Ras gets overexpressed. Actually Cao., et al. [26], in 2015 demonstrated that it hampers human gastric carcinoma AGS cells proliferation via downregulation of Ras proteins whereas up regulation of extracellular signal -regulated kinase (ERK1/2) [26]. Besides that Curcumin had the capacity to hamper along with regulation of ERK1/2 that result in downregulation of PI3K/Akt signaling pathway in cancer models $[27,28]$. Similarly targeting the Wnt/ $\beta$-catenin signaling pathway is an attractive strategy for cancer therapeutic application. Actually Wnt/ $\beta$-catenin signaling pathway overexpression is supposed to influence human cancers, with Curcumin possessing the capacity to stimulate cell cycle arrest at G2/M phase via manipulation of Wnt/ $\beta$-catenin signaling pathway. Dou., et al. [29], further documented that Curcumin can hamper Wnt/ $\beta$-catenin signaling pathway suppression through miR130a.

With the anticancer actions, Curcumin has the ability to target various cancer transcription factors. Numerous studies illustrated that Curcumin can block NFkB, along with AP1, transcription factor families [30]. Marquardt., et al. [31], evaluated the action of curcumin sensitive in addition to curcumin resistant liver cancer lines. Marquardt., et al. [31], sawthat in Curcumin sensitive cancer, there was hampering of NFKB, whereas in Curcumin resistant cells it was not so, instead they had sustenance of NFKB action. In case of lung cancer cells, curcumin resulted in reduction in the expression of proliferating cell nuclear Antigen (PCNA), phosphatidyl inositide 3-kinase (PI3K) as well as NFKB [30]. The sensitivity of human as well as rat glioma cells to radiation escalated subsequent to Curcumin therapy, with both expression of NFKB, along with AP1 got hampered [32]. Further Curcumin was demonstrated to cause reduction of expression amounts of STAT3-controlled CyclinD1. BCL2, Bcl-xL in pancreatic cancer cells [33]. Shanmugan., et al. [32], in
2015, further illustrated that Curcumin could hamper IL-6 stimulated STAT3- phosphorylation, besides STAT3nuclear translocation in case of multiple myeloma. Further more curcumin had the capacity to hamper cell proliferation, migration as well as invasion, yet facilitated apoptosis in retinoblastoma cells with antitumor actions occurring through miR99a as well as hence the hampering of JAK/STAT pathway.

\section{Hepatoshielding action}

Various agents like alcohol, drugs, pollutants, parasites as well as dietary constituents, besides obesity, DM as well as others can stimulate acute as well as chronic hepatic injuries that are liver fibrosis, non alcoholic steatohepapititis (NASH), non alcoholic fatty liver disease (NAFLD), in addition to cirrhosis [34].

Choudhary., et al. [35], showed that a Curcumin injection (8.98 $\mu \mathrm{m}$ ) in Swiss albino rats carbon tetrachloride -mediated oxidative hepatocellular damage resulted in reduction of NADPH-oxidase amounts as well as escalated the GR along with GST amounts as well as succinate dehydrogenase action [35]. For equivalent kind of hepatotoxicity, Curcumin delivery in Sprague-Dawley rats (200 $\mathrm{mg} / \mathrm{kg}$ ) escalated the hepatic glutathione amounts, in addition to causing reduction in Lipid peroxidase amounts as well as actions of both alanine aminotransferase (ALT) as well as aspartate aminotransferase (AST) [36]. Thus Curcumin might be an attractive agent for avoidance of oxidative stress- associated liver conditions by resulting in reduction of ALT, AST, as well as alkaline phosphatase (AP), whereas escalated GST, GR, GPx, SOD, Catalase (CAT) as well as decreasing NO in addition to hampering ROS generation [37]. Moreover Badria., et al. [38], illustrated that Curcumin treatment escalated the endogenous anti oxidant amounts (ascorbic acid, GSH, SOD, CAT) in the liver and spleen of chronic iron overloaded rats [38]. In certain vases of drug stimulated hepatotoxicity like the one that is streptozocin induced liver damage as well as paracetamol induced liver mitochondrial changes Curcumin had the capacity of ameliorating these actions in mice. Afrin., et al. [39], observed that Curcumin delivery in Sprague-Dawley rats secondary to streptozocin induced Diabetes mellitus (DM), hampered TNF $\alpha$, IL-1 $\beta$, MAPK, in addition to apoptosis signal regulating kinase1 (ASK1) in liver tissues. In case of NASH stimulated by low dose streptozocin as well as high fat diet (HFD), Afrin., et al. [39], visualized that Curcumin therapy could result in reduction of Oxidative stress, inflammation as well as lipogenesis, as well as miti- 
A Plethora of Actions of Curcumin - A Magical Agent for Treatment of Wide Range of Diseases Varying from Neuroinflammatory Disease (AD, PD)-IBD to DM and CVD, NAFLD, NASH Along with Various Cancers - A Systematic Review

gated fibrosis along with $\mathrm{HMGB}_{1}$. NFKB translocation in addition to signaling with regards to paracetamol induced liver mitochondrial changes, curcumin delivery attenuated mitochondrial impairment by hunting free radicals, stimulation of anti oxidant enzyme expression, as well as hampered NFKB action as well as transient receptor potential melastatin 2 (TRPM2) channels [40].

In mice with alcoholic fatty liver disease, Curcumin delivery ameliorated hepatocyte necroptosis, suppressed the ethanol stimulated pathway, hampered glyoxalate, dicarboxylate as well as pyruvate metabolism, modulated anti oxidant signaling pathway as well as upregulated detoxifying genes expression through the ERK/p-38/MAPK pathway [41].

It has further been illustrated that Curcumin delivery can ameliorate liver fibrosis along with cirrhosis $[15,42]$. Curcumin delivery in Sprague-Dawley rats with CCL4 -stimulated hepatic fibrosis that resulted in reduction in liver fibrosis via. i) reduction in extracellular matrix (ECM) over generation in hepatic stellate cells (HSCs) ii) disturbing platelet derived growth factor receptor (PDGFR)/ERK as well as mammalian target of rapamycin (mTOR) pathwayiii) activation of) Peroxisome Proliferator Activated Receptor $\gamma$ (PPAR $\gamma$ ) iv) upregulated PTEN as well as miR $29 \mathrm{~b}$ expression as well as v) down regulation of cannabinoid Receptors (CBR) type I as well as DNA methyltransferase3b (DNMT3b) [15,43].

\section{Cardioshielding action}

Cardiovascular diseases are believed to be a global human health problem, that escalates morbidity as well as mortality rates. As per various studies Curcumin is efficacious in avoidance of CVD [44]. Figure 6 illustrates how mechanistically Curcumin works on CVD. Major CV advantages are associated with their shielding actions on Atherosclerosis, hypertrophy, heart failure (HF), aortic aneurysm, stroke, myocardial infarction (MI) as well as Diabetic CV complications [45] in the form of molecular targets. It is significant, that Curcumin fresults in activation of Nrf2 that causes HO-1 getting induced, that is implicated in the Cardioshielding actions as well as anti inflammatory actions against Oxidative stress [46]. Like Sunagawa., et al. [47], demonstrated that curcumin has a natural p300 histone acetyl transferase (HAT) inhibitory action, whereas Monfoulet., et al. [48], illustrated that curcumin can escalate endothelial working as well as result in reduction of TNF $\alpha$-stimulated monocyte in endothelial cells via hampering of NFKB inhibition. Moreover Yao., et al. [49], observed that Curcumin results in reduction of angiotensin II type IR (AT1R) expression, hence avoiding CVD. In effect Curcumin respectively reduces the binding ability of AT1R gene promoter with its specificity protein (SP1). Cao., et al. [50], showed that Curcumin alleviates chronic heart failure by escalating p38MAPK, JNK, as well as ASK1.

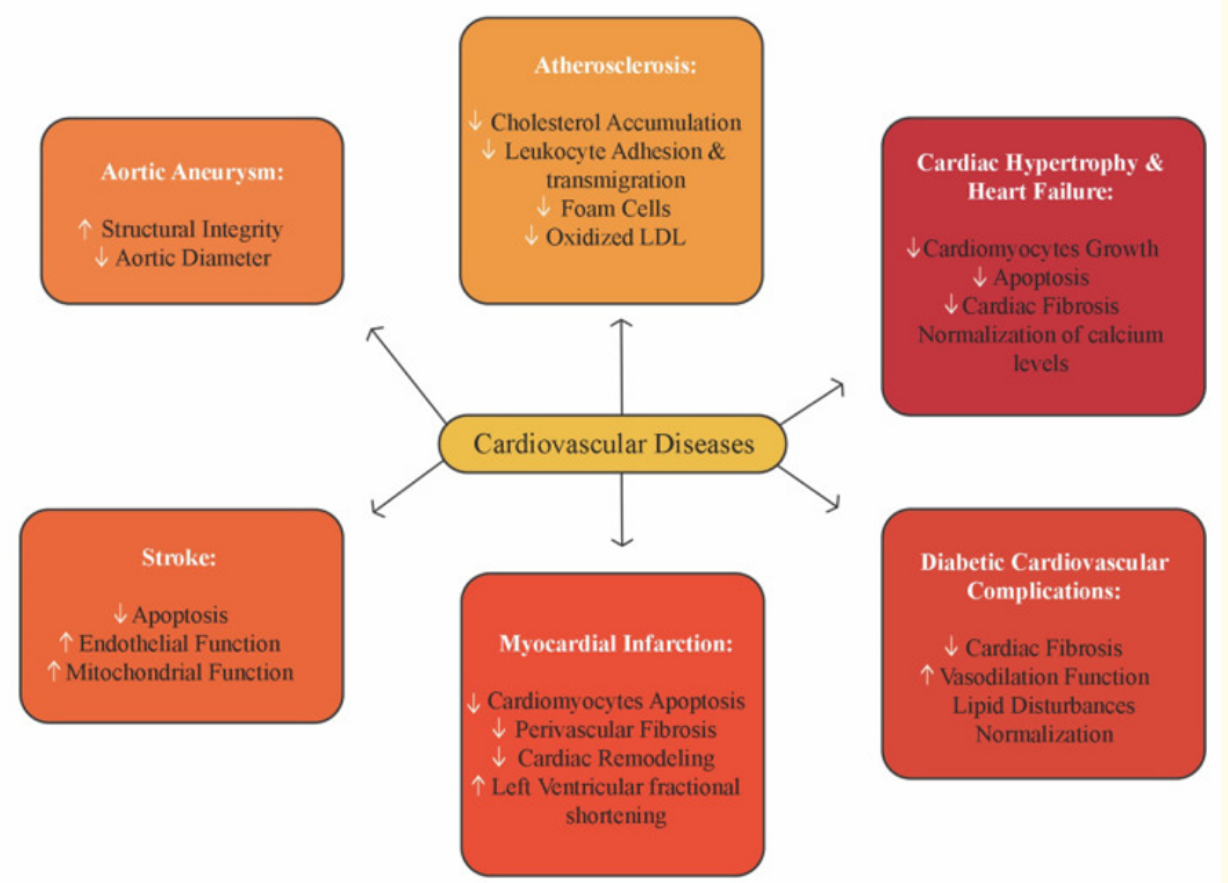

Figure 6: Courtesy ref no-2 - Curcumin action on cardiovascular diseases. 
A Plethora of Actions of Curcumin - A Magical Agent for Treatment of Wide Range of Diseases Varying from Neuroinflammatory Disease (AD, PD)-IBD to DM and CVD, NAFLD, NASH Along with Various Cancers - A Systematic Review

\section{Conclusions}

Across the last $50 \%$ of century great number of clinical trials have been performed to check the effectiveness, safety in addition to pharmacokinetics [51]. Curcumin was delivered in various kind of formulations like, capsules, tablets, powder nanoparticles, liposomal encapsulation as well as emulsions, with dose escalation studies documenting that Curcumin is safe in as great doses as $12 \mathrm{~g} /$ day for $3 \mathrm{mths}$. The biggest limitation had been the bioavailability, as well as to get over this problem newer Curcumin's nanomedicine formulations have been generated for enhancing the targeting of Curcumin effectiveness of pharmacokinetics, efficiency in addition to cellular uptake $[13,45]$. Its pleiotropic actions include a lot of inflammatory diseases like cancer, CVD, arthritis, atherosclerosis, DM, gastric disease, inflammatory bowel disease (IBD), psoariasis, HIV etc. On the same hand, a broad variety of molecular targets like proinflammatory cytokines, apoptotic proteins, NFKB, COX-2, -5, LOX, STAT3, CRP, PGE2, prostate specific antigen, intercellular cell adhesion molecule [ICAM]-1 as well as other adhesion molecule, phosphorylase kinase, in addition to transforming growth factor beta (TGF- $\beta$ ), triglycerides, ET-1, creatinine, HO-1, AST, ALT, that definitely calls for its multiple health actions. Intriguingly the future applications are evaluating for Curcuminstimulated cognitive actions. Actually Curcumin is accepted to be a molecule having the capacity to avoid/slowdown the pathological events resulting in age - associated dementia, cognitive decrease, or depression, although controversial conclusions that come out with certain doubts with regards to its efficiency. A recent paper evaluated the Clinical trials on Curcumin application in cognitive functions for evaluation of its actual efficacy [52]. Unluckily only 6 articles satisfied the inclusion criteria, hence the data was not enough to yield a precise evaluation of its results in various persons. Nevertheless, outcomes demonstrated that Curcumin is safe as well as well tolerated along with appears to be of greater use in mitigation of cognitive aberrations in elderly cases act AD as well as schizophrenia - associated symptoms. Additionally, various evaluations have pointed that Curcumin is a potential chemo avoiding as well as anticancer treatment in case of human papilloma virus (HPV) infection, in addition to primary as well as malignant squamous cell cancer [53]. Nevertheless, greater quality clinical trials are required to validate these advantageous actions of Curcumin in various Clinical situations. In that direction the Clinical Trial registered with the No-NCT02944578 is enrolling women with high grade squamous intraepithelial cervcal lesions (http://clinicaltri- als.gov). Furthermore, curcumin avoids the generation of renal injury in the streptozotocin induced type 1 Diabetic rats which got correlated with decreased amounts of renal H3 acetylation. Subsequently, it got demonstrated that the Curcumin analog C66 inhibits HAT p300/CBP action as well as subsequent histone acetylation in streptozotocin induced Diabetic mice avoiding the expression of renal fibrotic genes. Another p300/CBP inhibitor C646 inhibited TGF $\beta 1$ - stimulated epithelial -mesenchymal -transition (EMT) of human peritoneal mesothelial cells getting exposed to escalated glucose situation via manipulation of H3 acetylation [54]. In view of these limitations on Oral delivery of curcumin (CUR) with its restricted efficacy in view due to CUR's poor systemic bioavailability secondary to its first-pass metabolism along with low solubility Lim as well as Hadinoto advocated Buccal delivery of CUR nanoparticles can take care of the poor bioavailability issue by prevention of first-pass metabolism along with solubility escalation that gets allowed by CUR nanoparticles. Buccal film administration of drug nanoparticles, however, has been restricted to low drug payload. Thus, Lim as well as Hadinoto evaluated the feasibilities of three mucoadhesive polysaccharides, i. e., hydroxypropyl methylcellulose (HPMC), starch, and hydroxypropyl starch as buccal films of amorphous CUR-chitosan nanoplex at high CUR payload. Both HPMC and starch films could allow high CUR payload without side effects on the films' properties. Starch films far superior CUR liberation profiles at high CUR payload in view of rapid break down time of starch films lowered the precipitation chances of the highly supersaturated CUR concentration generated by the nanoplex. In Contrast to unmodified starch, hydroxypropyl starch films exhibited superior CUR release, with sustained release of about $100 \%$ of the CUR payload in 4h. Hydroxypropyl starch films also demonstrated good payload uniformity, minimal weight/thickness variations, high folding endurance, and good long-term storage stability. Thus hydroxypropyl starch might be suitable mucoadhesive polysaccharide for high-payload buccal film applications in addition to aid in utilization of curcumin overcoming those limitations cited earlier [55].

\section{Bibliography}

1. Milobedzka J., et al. "Zur Kenntnis des Curcumins". Berichte der deutschen chemischen Gesellschaft 43 (1910): 2163-2170.

2. Sharifi -Rad., et al. "Turmeric and its major compound Curcumin on health: bioactive effects and safety profiles for food, Pharmaceutical, Biotechnological, and medicinal applications". Frontiers in Pharmacology 11 (2020): 01021. 
3. Yeung AWK., et al. "Curcumin: Total scale analysis of the scientific literature”. Molecules 24 (2019): 1393.

4. Xu XY., et al. "Bioactivity, health benefits, and related molecular mechanisms of Curcumin: current progress, challenges and perspectives". Nutrients 10 (2018): 1553.

5. Priyadarsini KL., et al. "Role of phenolic-O-Hand methylene hydrogen on the free radical reactions and antioxidant activity of curcumin". Free Radical Biology and Medicine 35 (2003): 475-484.

6. NardoL., et al. "Role of H-bond formation in the photo reactivity of curcumin”. Spectroscopy 22 (2008): 187-198.

7. AK Tuba and GulcinI. "Anti-oxidant and radical scavenging properties of curcumin". Chemico-Biological Interactions 174 (2008): 27-37.

8. Dai W., et al. "Curcumin provides neuroprotection in model of traumatic brain injury via the Nrf2-ARE signaling pathway". Brain Research Bulletin 140 (2018): 65-71.

9. Edwards RL., et al. "The anti-inflammatory activity of Curcumin is mediated by its Oxidative Metabolites". Journal of Biological Chemistry 292 (2017): 21243-21252.

10. Aggarwal BB and Sung B. "Pharmacological basis for the role of Curcumin in chronic disease: an age-old spice with modern targets". Trends Pharmacology Science 30 (2009): 85-94.

11. Yang H., et al. "Curcumin enhances anticancer effects of 5-fluorouracil against gastric cancer through down regulation of COX2 and NFKB signaling pathways". Journal of Cancer 8 (2017): 3697-3706.

12. Teter B., et al. "Curcumin restores innate immune, Alzheimer's disease risk gene expression to ameliorate Alzheimer's pathogenesis". Neurobiology of Disease 127 (2019): 432-448.

13. Salehi B., et al. "Curcumin's nanomedicine formulations for therapeutic application in Neurological disease". Journal of Clinical Medicine 9.2 (2020a): 430.

14. Cianciulli A., et al. "PI3K/Akt signaling pathway plays a crucial role in the anti-inflammatory effects of Curcumin on LPS activated microglia". International Immuno Pharmacology 36 (2016): 282-290.

15. Chen N., et al. "Suppression of the TGF-beta/Smad signaling pathway and inhibition of hepatic stellate cells proliferation play a role in the hepatoprotective effects of Curcumin against alcohol induced hepatic fibrosis". International Journal of Molecular Medicine 34 (2014): 1110-1116.
16. Goozee KG., et al. "Examining the Potential Clinical value of Curcumin in the prevention and diagnosis of Alzheimer's disease". British Journal of Nutrition 115 (2016): 449-465.

17. Ganesh P., et al. "A Potential role of periodontal inflammation in Alzheimer's disease: A review". Oral Health and Preventive Dentistry 15 (2017): 7-12.

18. Qureshi M., et al. "Therapeutic Potential of Curcumin for multiple sclerosis". Neurological Sciences 39.2 (2018): 207-214.

19. Yance DRJR and Sagar SM. "Targeting angiogenesis with integrative cancer Therapies". Integrative Cancer Therapies 5 (2006): 9-29.

20. Astinfeshan M., et al. "Curcumin inhibits angiogenesis in endothelial cells using down regulation of the PI3K/Akt signaling pathway". Food Bioscience 29 (2019): 86-93.

21. Kandoth C., et al. "Mutational landscape and significance across 12 major cancer types". Nature 502 (2013): 332-339.

22. Hermeking H. "p53 enters the microRNA world". Cancer Cell 12 (2007): 414-418.

23. Ye M., et al. "Curcumin promotes apoptosis by activating the p53- miR 192-5p/215-XIAP pathway in non-small cells lung cancer". Cancer Letters 357 (2015): 196-205.

24. Watson JL., et al. "Curcumin causes super oxide anion production and p53- independent apoptosis in human colon cancer cells". Cancer Letters 297 (2010): 1-8.

25. Chiu TL and Su CC. "Curcumin inhibits proliferation and migration by increasing the $\mathrm{Bax}$ to $\mathrm{Bcl} 2$ ratio and decreasing nuclear factor $\kappa B p 65$ expression in breast cancer MDA MB231 cells". International Journal of Molecular Medicine 23 (2009): 469-475.

26. Cao Q., et al. "Ras/ERK signaling pathway is involved in Curcumin induced cell cycle arrest and apoptosis in human gastric carcinoma AGS cells". Journal of Asian Natural Products Research 17 (2015): 56-63.

27. Rana C., et al. "Downregulation of PI3K/Akt/PTEN pathway and activation of mitochondrial intrinsic apoptosis by diclofenac and Curcumin in colon cancer". Molecular and Cellular Biochemistry 402 (2017): 225-241.

28. Kasi PD., et al. "Molecular targets of Curcumin for cancer therapy: an updated review". Tumor Biology 37 (2016): 1301713028. 
29. Dou., et al. "Curcumin suppresses the colon cancer proliferation by inhibiting Wnt/ $\beta$-catenin signaling pathway via miR130a". Frontiers in Pharmacology 8 (2017): 877.

30. Man S., et al. "Curcumin enhances the anti-cancer effects of Paris Saponin II in lung cancer cells". cell proliferation 51 (2018): e12458.

31. Marquardt JU., et al. "Curcumin effectively inhibits oncogenic nuclear factor Kappa B signaling and restrains stemness features in liver cancer". Journal of Hepatology 63 (2015): 661669.

32. Shanmugan MK., et al. "The multifaceted role of curcumin in cancer prevention and treatment". Molecules 20 (2015): 27282769.

33. Rajitha B and Nagarajan GP. "Curcumin and genistein role in regulation of STAT3- in pancreatic cancer. In role of transcription factors in Gastrointestinal malignancies". Eds GP Nagaraju, PV Bramhachari (Singapore: Springer Singapore) (2017): 427-435.

34. Macias-Perez JR., et al. "Curcumin and $\alpha / \beta$ adrenergic antagonist co treatment reverses liver cirrhosis in hamsters: participation of Nrf2 and NFKB". Journal of Immunology Research (2019): 3019794-3019794.

35. Choudhary ST., et al. "Vesicular (liposomal and nanoparticulated) delivery of curcumin: a comparative study on carbon tetrachloride-mediated Oxidative Hepatocellular damage in rat model". International Journal of Nanomedicine 11 (2016): 2179-2193.

36. Lee HY., et al. "Turmeric extract and its active compound, curcumin, protect against chronic CCL4- induced liver damage by enhancing anti oxidation". BMC Complementary and Alternative Medicine 16 (2016): 316.

37. Farzaei MH., et al. "Curcumin in liver diseases: a systematic review of the cellular mechanisms of Oxidative stress and Clinical perspective". Nutrients 10.7 (2018): 855.

38. Badria FA., et al. "Curcumin attenuates iron accumulation and Oxidative stress in the liver and spleen of chronic iron overloaded rats". PLoS One 10 (2015): e13456.

39. Afrin R., et al. "Curcumin ameliorates streptozocin induced liver damage through modulation of endoplasmic reticulum stress mediated apoptosis in Diabetic rats". Free Radical Research 49 (2015): 279-289.
40. Granados-Castro LF., et al. "Curcumin prevents paracetamol induced liver mitochondrial alterations". Journal of Pharmacy and Pharmacology 68 (2016): 245-256.

41. Gou M., et al. "Curcumin loaded biodegradable polymeric micelles for colon cancer therapy in vitro and in vivo". Nanoscale 3 (2011): 1558-1567.

42. Zhong W., et al. "Curcumin alleviates lipopolysaccharide induced sepsis and liver failure by suppression of Oxidative stress related inflammation via PI3K/AKT and NF-Kappa B- related signaling". Biomedicine and Pharmacotherapy 83 (2016): 302-313.

43. Zhang F., et al. "Curcumin attenuates angiogenesis in liver fibrosis and inhibits angiogenic properties of hepatic stellate cells". Journal of Cellular and Molecular Medicine 18 (2014): 1392-1406.

44. Li H., et al. "Curcumin, the golden spice for treating cardiovascular diseases”. Biotechnology Advances 38 (2019): 107343.

45. Salehi B., et al. "Therapeutic applications of Curcumin's nanomedicine formulations in cardiovascular diseases". Journal of Clinical Medicine 9.3 (2020b): 746.

46. Pittala V., et al. "Effects of polyphenolic derivatives in hemoxygenase system in metabolic dysfunction". Current Medicinal Chemistry 25 (2018): 1577-1595.

47. Sunagawa Y., et al. "Curcumin and its demothoxy derivatives possess p300HAT inhibitory activity and suppress hypertrophic responses in cardiomyocytes". Journal of Pharmacological Sciences 136 (2018): 212-217.

48. Monfoulet LE., et al. "Curcumin modulates endothelial permeability and monocyte trans endothelial migration by affecting endothelial cell dynamics". Free Radical Biology and Medicine 112 (2017): 109-120.

49. Yao Y., et al. "Curcumin exerts its antihypertensive effect by down regulating the AT1 receptor Vascular smooth muscle cells". Scientific Reports 6 (2016): 25579.

50. Cao O., et al. "Dickkopf3 upregulation mediates the cardioprotective effects of Curcumin on chronic heart failure". Molecular Medicine Reports 17 (2018): 7249-7257.

51. Subramani PA., et al. "Clinical trials of Curcumin, camptothecin, astaxanthine and biochanin". Natural product-derived compounds in clinical trials 136 (2018): 212-217. 
52. Zhu LN., et al. "Curcumin Intervention for cognitive functions in different types of people: a systematic review and metaanalysis". Phytotherapy Research 33.3 (2018): 524-533.

53. Teymouri M., et al. "Curcumin as a multifaceted compound against human papilloma virus infection and cervical cancers: a review of chemistry, cellular molecular, and pre-Clinical features". Bio Factors 43 (2017): 331-346.

54. Kulvinder Kochar Kaur., et al. "Potential role of Epigenetic Modulation in prevention or therapy for Diabetic Kidney Disease-still a dream or a reality-A Systematic Review". Diabetic Nephropathy and Diabetes Management 1:1 (2021): 1-26.

55. Lim KM and Hadinoto K. "High payload buccal delivery system of amorphous curcumin- Chitosan Nanoparticle Complex in Hydroxypropyl Methylcellulose and Starch Films". International Journal of Molecular Sciences 22.17 (2019): 9399.

\section{Assets from publication with us}

- Prompt Acknowledgement after receiving the article

- Thorough Double blinded peer review

- Rapid Publication

- Issue of Publication Certificate

- High visibility of your Published work

Website: www.actascientific.com/

Submit Article: www.actascientific.com/submission.php

Email us: editor@actascientific.com

Contact us: +919182824667 